\title{
Facial selectivity in the addition of ketene acetals to nitrones towards chiral non-racemic isoxazolidin-5-ones. A semiempirical study
}

\author{
Pedro Merino* and Juan A. Mates \\ Laboratorio de Sintesis Asimetrica. Departamento de Quimica Organica. Facultad de Ciencias. \\ Universidad de Zaragoza. E-50009 Zaragoza. Aragon. Spain. \\ E-mail:pmerino@posta.unizar.es
}

This manuscript is dedicated to Prof. B. S. Thyagarajan

(received 18 Mar 01; accepted 18 Sep 01; published on the web 26 Sep 01)

\begin{abstract}
A semiempirical study (PM3) of the addition of silyl ketene acetals to D-glyceraldehyde derived nitrones has been carried out in order to rationalize the observed diastereofacial selectivity. Activation with Lewis acid must be considered in the calculations to achieve concordant results. Thus, the addition to trimethylsilyl trifluoromethane sulfonate-activated nitrones, leading to the preferential formation of syn adducts, may be explained by a stepwise mechanism. On the other hand, the diastereofacial anti selectivity observed in the addition to diethylaluminium chlorideactivated nitrones can be interpreted in terms of a concerted mechanism.
\end{abstract}

Keywords: Isoxazolidines, nitrones, ketene acetals, semiempirical calculations

\section{Introduction}

Optically active isoxazolidines are important synthetic intermediates that can be used for preparing a variety of nitrogenated natural products and derivatives. ${ }^{1}$ Their synthetic utility mainly resides in the fact that they can be reduced under mild conditions to give 1,3aminoalcohols. In recent years, a number of reports have originated from several laboratories ${ }^{2}$ concerning the construction of chiral non-racemic isoxazolidines as convenient precursors of a new class of nucleoside analogues. ${ }^{3}$

In particular, 3,4-disubstituted isoxazolidin-5-ones $\mathbf{1}$ are the key intermediates in the 
synthesis of isoxazolidinyl nucleosides. ${ }^{2-4}$ Typically, compounds $\mathbf{1}$ are produced from the 1,3dipolar cycloaddition of a ketene and a nitrone (Scheme 1). Alternatively, the addition of a ketene acetal can be employed for the construction of the heterocyclic moiety after intramolecular cycloaddition of the intermediate hydroxylamine. ${ }^{4}$

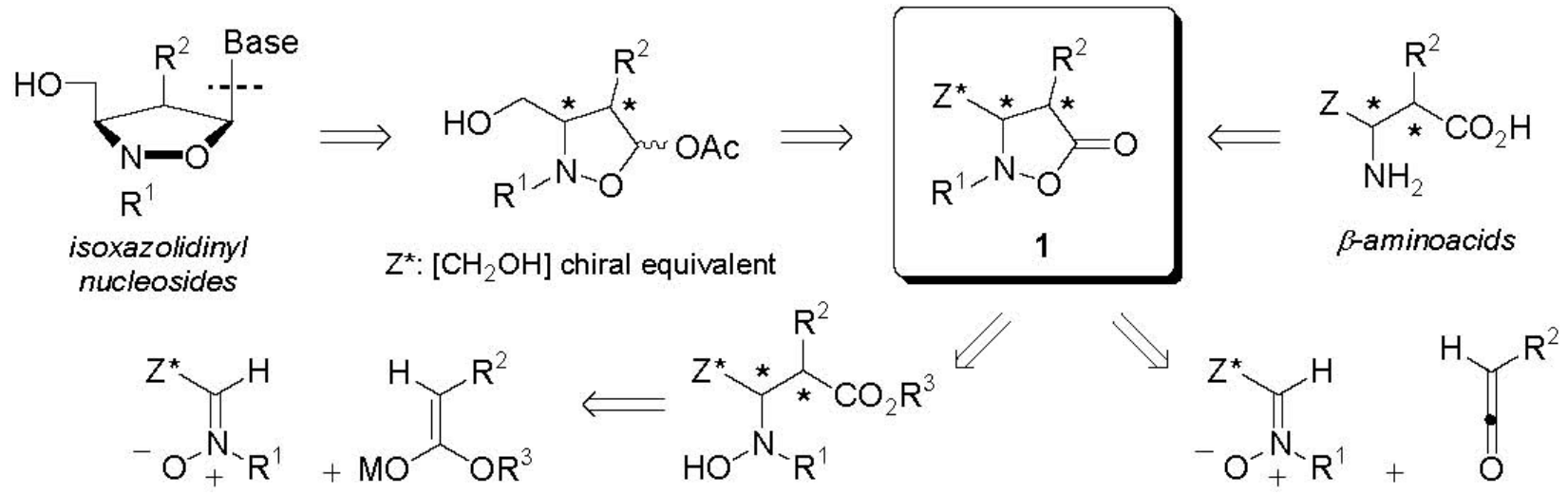

\section{Scheme 1}

The addition of ketene acetals to chiral nitrones has been scarcely investigated. ${ }^{5}$ Compared to other metal-derived ketene acetals, the silyl derivatives requires the use of Lewis acids as activating agents ${ }^{6}$ and can be applied to the stereoselective synthesis of isoxazolidines. ${ }^{7}$ In this context, we have recently reported the addition of silyl ketene acetals 3 to D-glyceraldehyde derived nitrones 2 to give isoxazolidin-5-ones 1. (Scheme 2). ${ }^{7}$ The reaction afforded a complex mixture of hydroxylamines and cyclic compounds that can be transformed, in a one-flask procedure, to the targeted isoxazolidin-5-ones $\mathbf{1}$. The selectivity of the reaction can be controlled by choosing the adequate Lewis acid. 


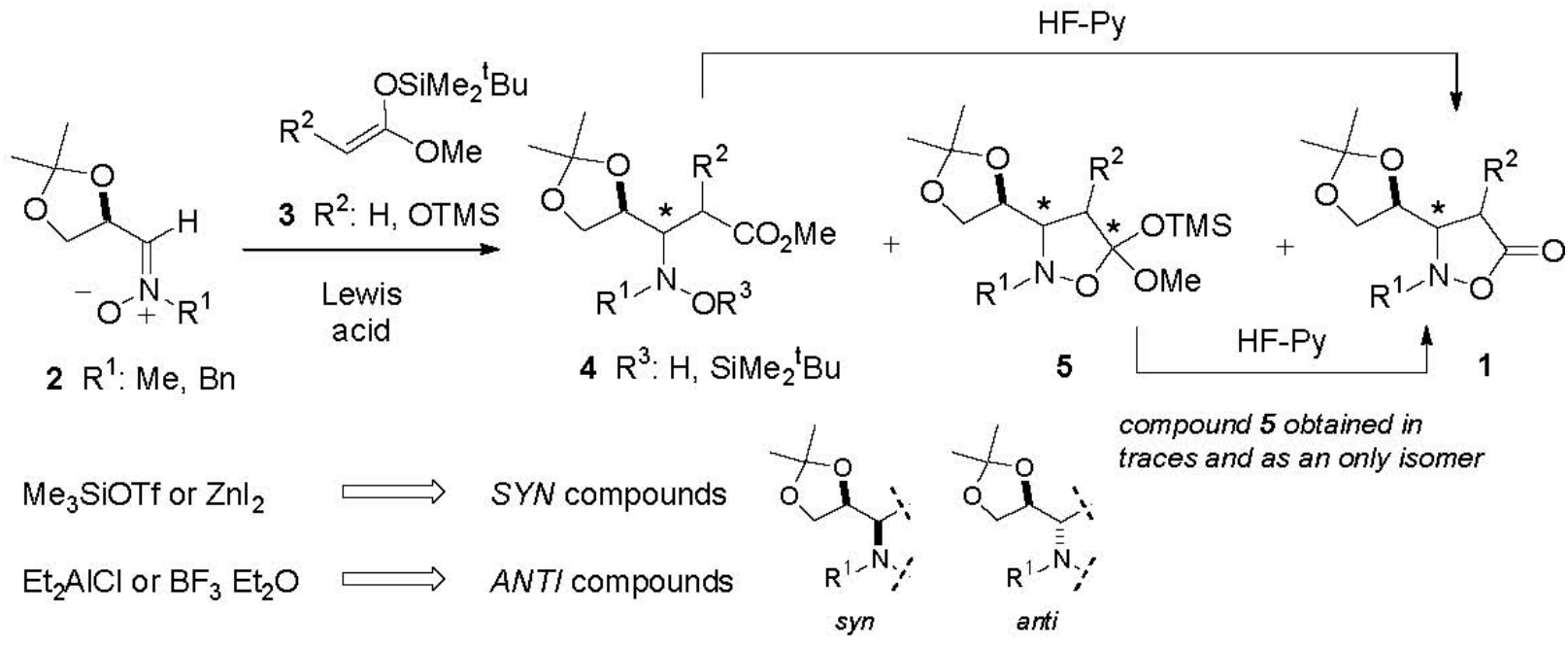

\section{Scheme 2}

Thus, whereas activation with trimethylsilyl trifluoromethane sulfonate (TMSOTf) led to syn adducts, the use of diethylaluminium chloride (DEAC), led to anti compounds preferentially. It is worth noting that in some instances traces of the cyclic compound $\mathbf{5}$ were also observed.

Now, we wish to report a computational study of the difference between addition reactions of ketene acetals to nitrone $\mathbf{2}$ without activation and activated by rather different additives (TMSOTf and DEAC).

\section{Computational methods}

Semiempirical methods are intended for studying large and complicated molecular systems of interest for which an application of ab initio methods is generally prohibitive in terms of computational effort. Furthermore, when several mechanisms can be invoked for related processes the problem of determining the operating mechanism in each case can be most conveniently and economically tackled following a semiempirical approach at the first stages of the computational study, considering the limitations of the underlying approximations. ${ }^{8}$ All calculations were performed with the MOPAC package ${ }^{9}$ using the PM3 method. ${ }^{10}$ The potential energy surfaces (PESs) for the studied processes have been calculated in detail. Critical points have been located without any geometry restriction and have characterized through the calculation of the force constants matrix by ensuring that they correspond to minima or saddle points on the PES; i.e., they have zero or one and only one imaginary frequency, respectively. All the reaction paths have been checked by tracing the intrinsic reaction coordinate (IRC) ${ }^{11}$ from the transition structure to the lower energy structures it connects. Optimized geometries (in PDB format) of all stationary points are available from the authors. 


\section{Results and Discussion}

\section{Without Lewis acids}

Several mechanisms can be proposed for the addition of silyl ketene acetals to nitrones. Greene et al. ${ }^{5 b}$ invoked a pentacoordinated silicon atom in the transition state, the $\mathrm{C}-\mathrm{C}$ bond being formed at an initial stage after which the silyl group is transferred to the hydroxyamino functionality. This mechanism is illustrated for nitrone $\mathbf{2}$ in Scheme 3.

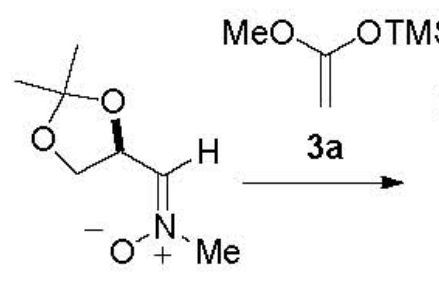

2

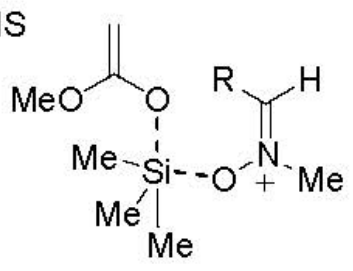

C1

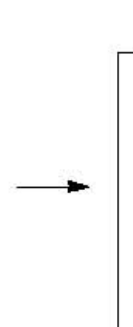

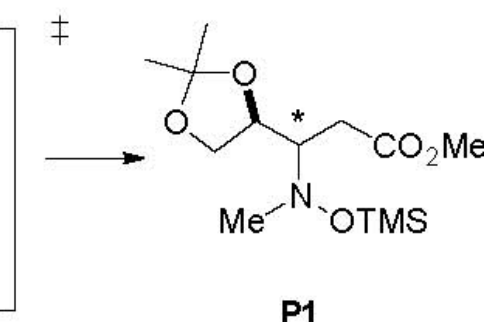

\section{Scheme 3}

We have investigated this mechanism with nitrone $2\left(\mathrm{R}^{1}=\mathrm{Me}\right)$ and silyl ketene acetal 3a $\left(\mathrm{R}^{2}\right.$ $=\mathrm{H})$ and we located two possible transitions states for Re (TS1-Re) and Si (TS1-Si) attacks which are shown in Figure 1. The IRC analysis showed as starting points the corresponding complexes $\mathbf{C 1}$ in which a pentacoordinated silicon atom had been formed. The energies $(\mathrm{kcal} / \mathrm{mol})$ of the corresponding stationary points are given in Table 1 . The products are the most stable species (3-10 Kcal/mol below the reagents) and the most favorable pathway is that corresponding to the Re attack -which leads to the syn compound-. 

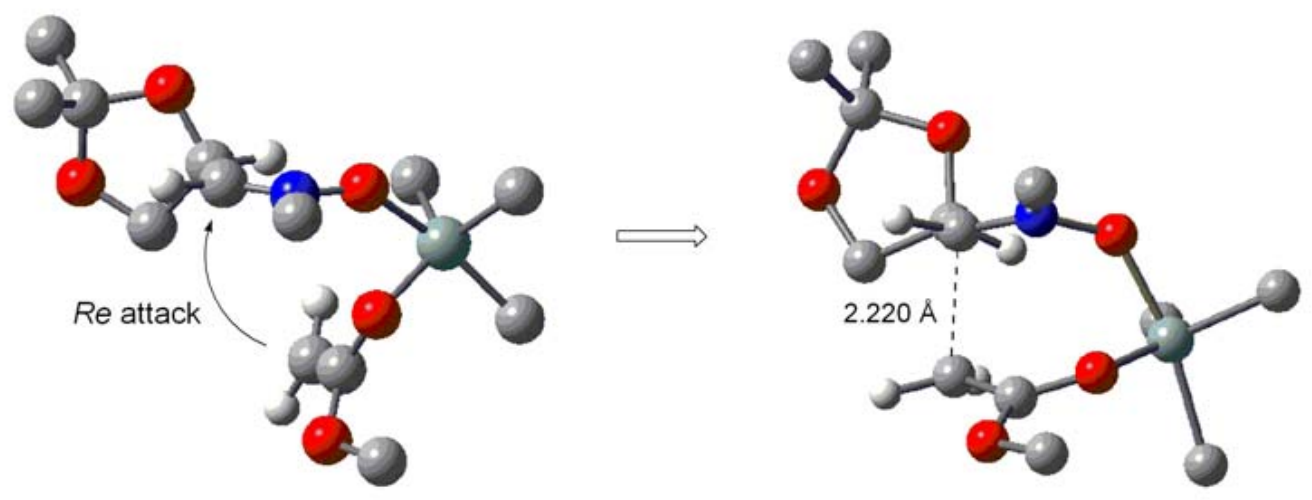

TS1-Re
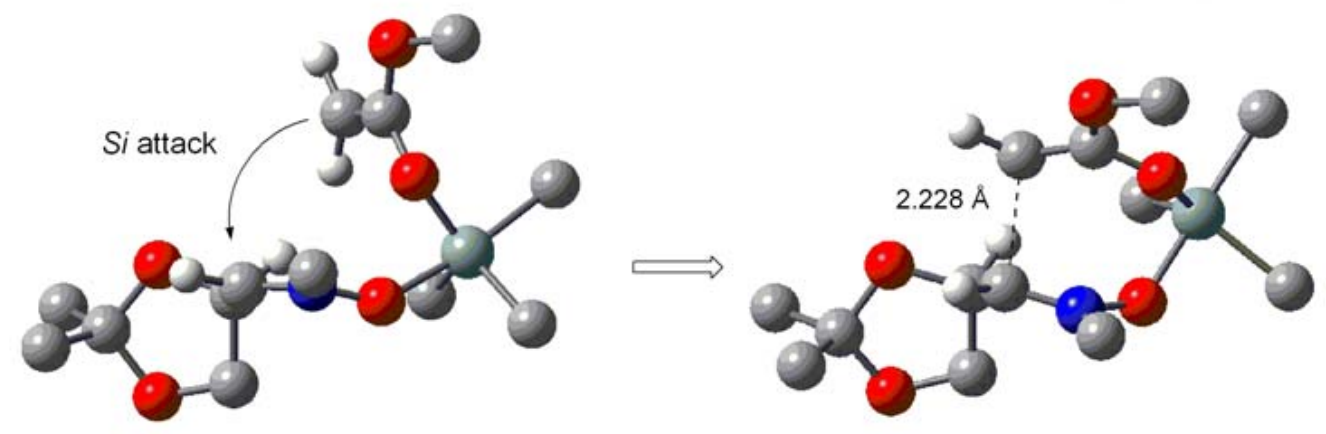

TS1-Si

Figure 1. Starting complexes and transition structures for the pentacoordinated-silicon mechanism.

However, the positive values of energy found for the formation of the starting complexes C1 indicate that the process is not favoured at all. This hypothesis is also confirmed by the high activation energy (36.7 and $37.4 \mathrm{Kcal} / \mathrm{mol}$ for TS1-Re and TS1-Si, respectively) found for the formation of the transition states.

An identical mechanism can also applied to the nucleophilic addition of lithium and sodium ketene acetals to nitrones previously reported by us. ${ }^{7}$ Also in this case the reaction starts with the formation of the corresponding complexes $\mathbf{C 2}$ without energy barrier (Scheme 4).

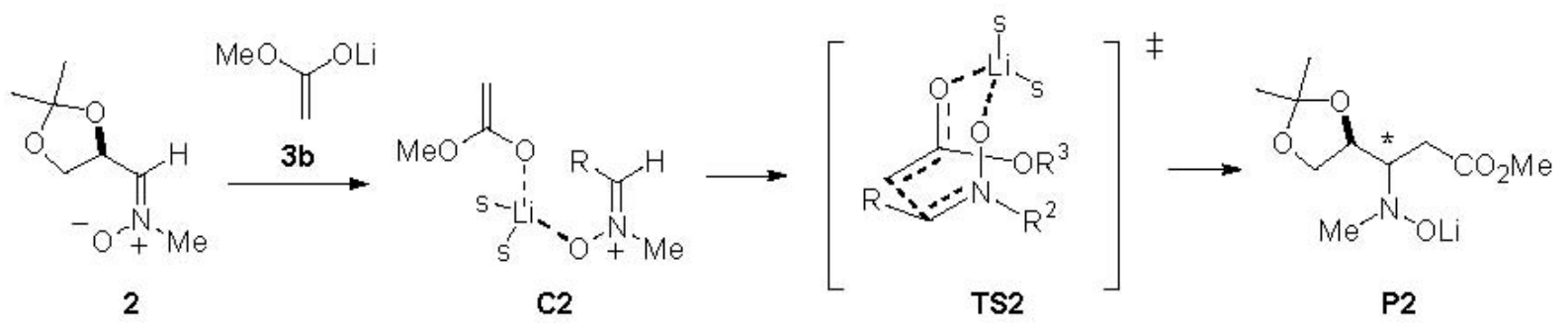

\section{Scheme 4}



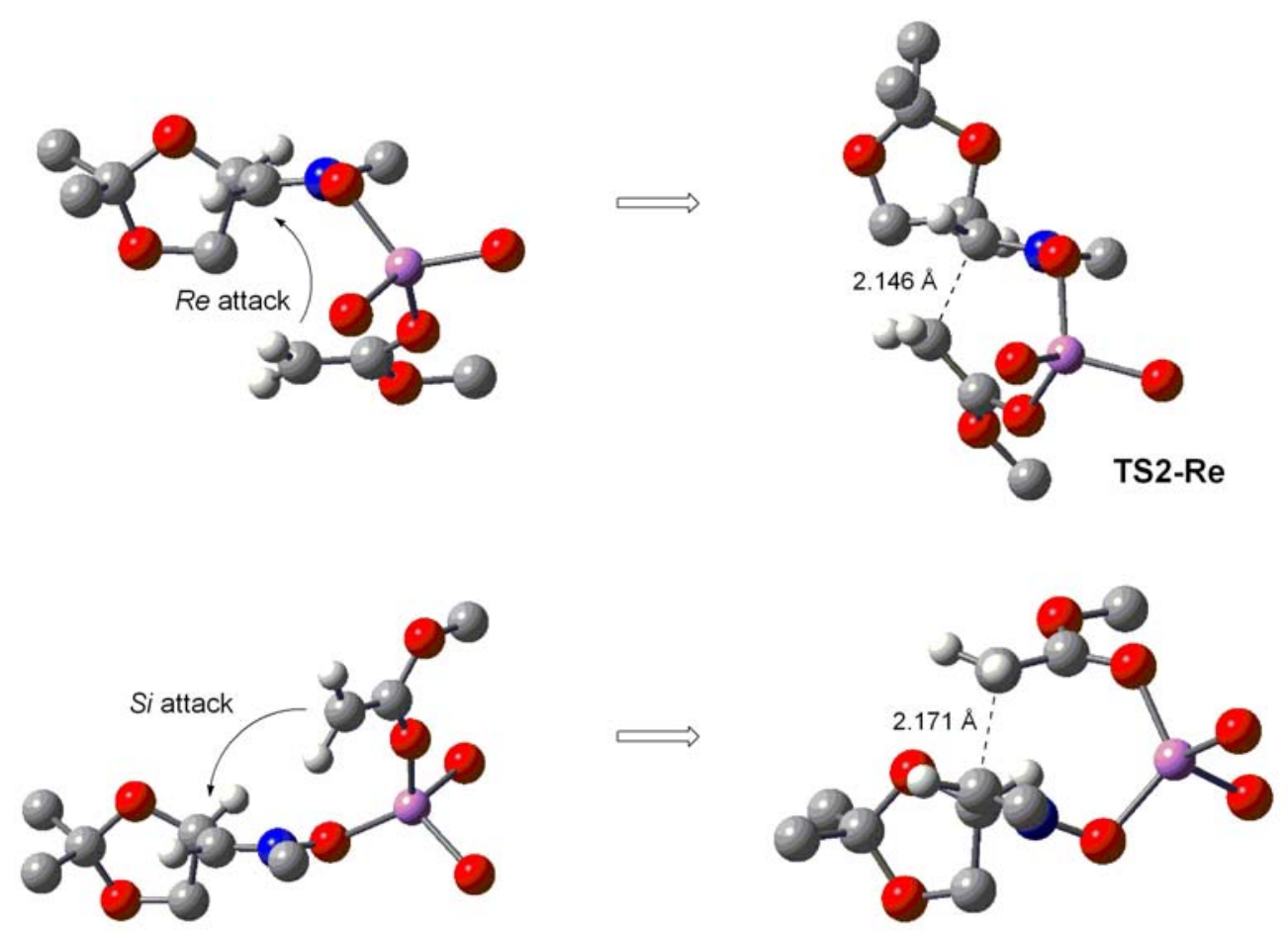

TS2-Si

Figure 2. Starting complexes and transition structures for addition of lithium ketene acetals.

Calculation of the corresponding reaction paths for the addition of a lithium ketene acetal afforded similar results. ${ }^{12}$ Two starting complexes and two transition structures TS2-Re and TS2-Si were located (Figure 2), the most stable being that corresponding to the Re- attack, in good agreement with the experimental results. The energy values for the stationary points are given in Table 1 . The energy profile for the addition of both silyl and lithium enolates is given in Figure 3. 
Table 1. Heats of formation (PM3) of the corresponding reagents, starting complexes, transition structures and products for the nucleophilic addition of silyl and lithium ketene acetal to 2 in the absence of Lewis acids

\begin{tabular}{ccc}
\hline & Energy (Kcal/mol) & Relative energy to reactants ${ }^{\mathrm{a}}$ \\
\hline $\mathbf{2}$ & -79.6 & \\
3a & -135.7 & \\
3b & -204.8 & \\
C1-Re & -197.2 & 18.1 \\
C1-Si & -197.0 & 18.4 \\
TS1-Re & -178.6 & 36.7 \\
TS1-Si & -177.9 & 37.8 \\
P1-syn & -226.2 & -10.8 \\
P1-anti & -219.2 & -3.8 \\
C2-Re & -295.0 & -10.6 \\
C2-Si & -295.7 & -11.3 \\
TS2-Re & -281.9 & 2.5 \\
TS2-Si & -278.6 & 5.7 \\
P2-syn & -305.9 & -21.5 \\
P2-anti & -310.3 & -25.9 \\
\hline
\end{tabular}

a The energy of the reactants has been calculated as the sum of that of the nitrone and the corresponding ketene acetal, i.e.: $-215.3 \mathrm{Kcal} / \mathrm{mol}$ for $\mathbf{2}$ and 3a and $-284.4 \mathrm{Kcal} / \mathrm{mol}$ for $\mathbf{2}$ and $\mathbf{3 b}$.

There are substantial differences between lithium and silyl ketene acetals. Whereas the former react with the nitrone in a straightforward way without any additive, the latter need of a Lewis acid as an activating agent. The formation of the starting complexes in the reaction with the silyl derivative is endothermic while the same process is exothermic in the case of the lithium derivative. As a consequence, the formation of starting complexes C2 is strongly favoured. In addition, rather lower activation energies are found for the reaction with the lithium enolate when compared with the reaction of the silyl enolate. So, it is possible to conclude that whereas the mechanism shown in Scheme 3 does not represent a realistic situation since no Lewis acids are considered, that represented in Scheme 4 corresponds to the experimentally observed process. 


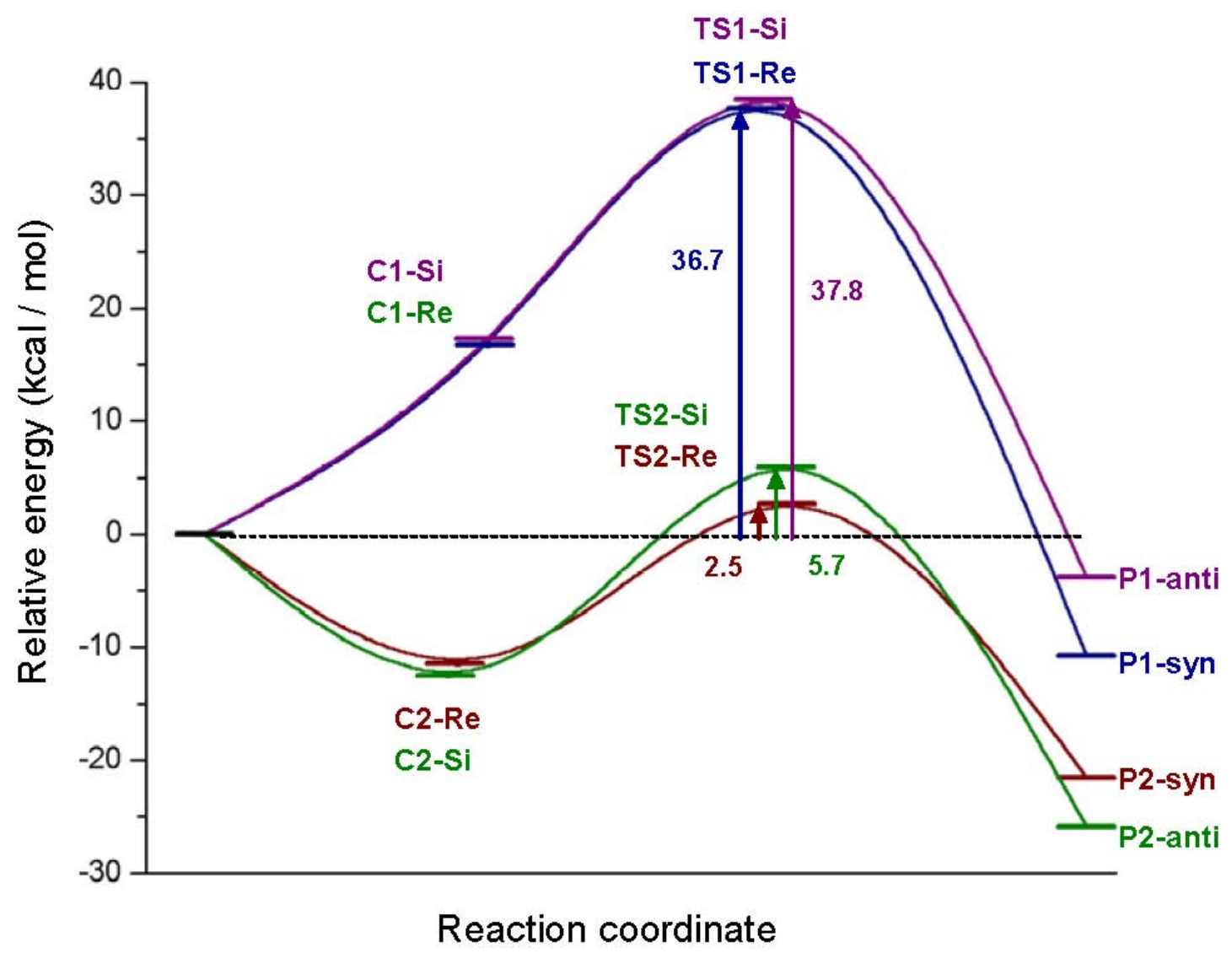

Moreover, the pentacoordinated-silicon mechanism cannot explain the formation of the orthoester 5 (Scheme 2), which seems to arise from a more concerted mechanism. In fact, it is possible to evaluate the formal 1,3-dipolar cycloaddition of nitrone $\mathbf{2}$ and silyl ketene acetal 3a to give orthoesters 5 (Scheme 5). For this process four transition states (TS3-Re-endo, TS3-Reexo, TS3-Si-endo and TS3-Si-exo) were located, since in addition to Re and Si attacks, endo and exo approaches should also be considered. (Figure 4).<smiles>C=C(OC)OCCCC(C1COC(C)(C)O1)=[N+](C)[O-]</smiles>

2<smiles>[R]C1=CC(OC)(O[Na])ON1C</smiles>

TS3<smiles>CO[C@@H]1C[C@@H]([C@@H]2COC(C)(C)O2)N(C)O1</smiles>

$5 \equiv \mathrm{P} 3$

\section{Scheme 5}




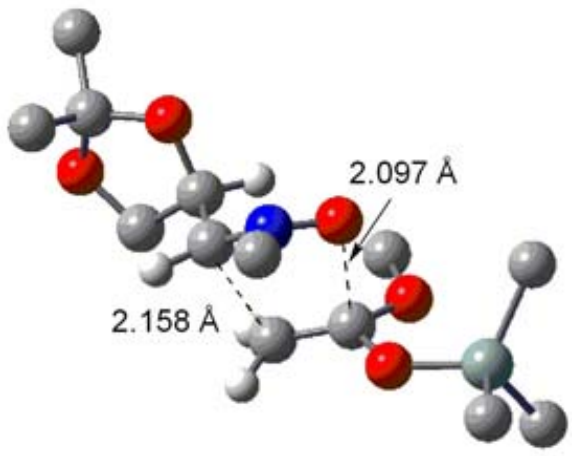

TS3-Re/endo

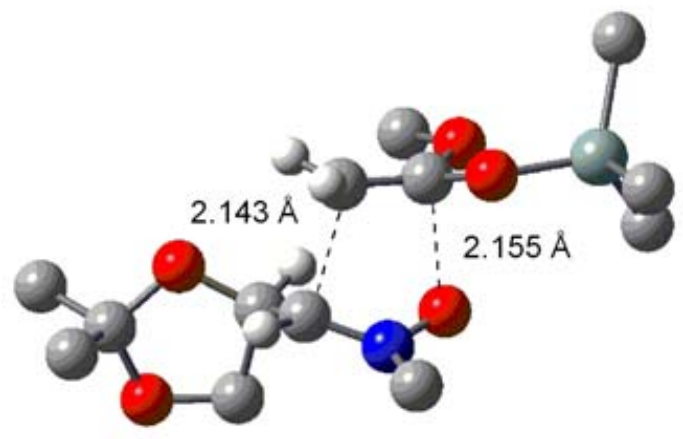

TS3-Si/endo

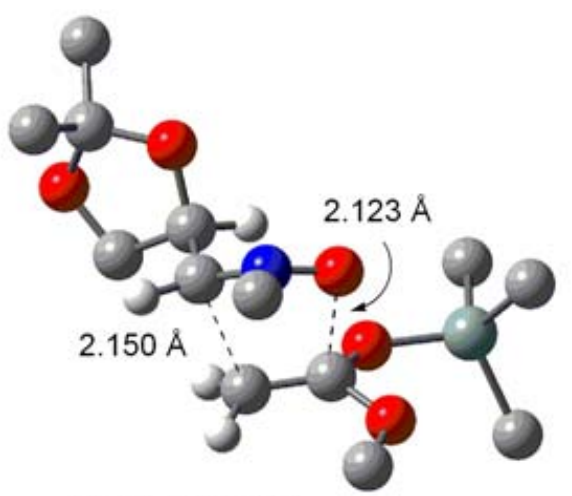

TS3-Re/exo

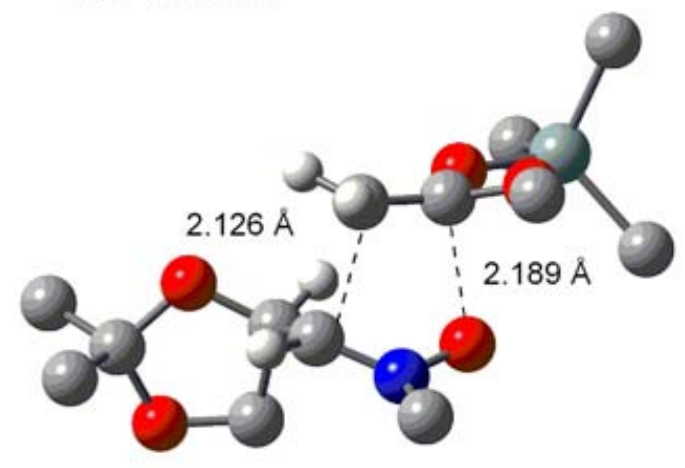

TS3-Si/exo

Figure 4. Transition states for the concerted reaction between 2 and 3a in the absence of Lewis acids.

The energy values for reactants, transition structures and diastereomeric products are given in Table 2 and the corresponding energy profile in Figure 5. In this case, the preferential formation of the syn adducts is well-predicted although very close values are obtained for the addition by the two diastereotopic faces. 
Table 2. Heats of formation (PM3) of the corresponding reagents, transition structures and products for the 1,3-dipolar cycloaddition of silyl ketene acetal 3a to 2 in the absence of Lewis acids

\begin{tabular}{ccc}
\hline & Energy $(\mathrm{Kcal} / \mathrm{mol})$ & Relative energy to reactants $^{\mathrm{a}}$ \\
\hline $\mathbf{2}$ & -79.6 & \\
3a & -135.7 & \\
TS3-Re-endo & -182.3 & 33.0 \\
TS3-Re-exo & -179.8 & 35.6 \\
TS3-Si-endo & -181.2 & 34.1 \\
TS3-Si-exo & -179.8 & 35.6 \\
P3-syn-trans $^{\mathrm{b}}$ & -251.6 & -36.3 \\
P3-syn-cis $^{\mathrm{c}}$ & -251.8 & -36.4 \\
P3-anti-trans $^{\mathrm{d}}$ & -253.0 & -37.7 \\
P3-anti-cis $^{\mathrm{e}}$ & -252.0 & -36.7 \\
\hline
\end{tabular}

${ }^{\mathrm{a}}$ The energy of the reactants has been calculated as the sum of that of the nitrone and the silyl ketene acetal, i.e.: $-215.3 \mathrm{Kcal} / \mathrm{mol} .{ }^{\mathrm{b}}$ (3R,5R) obtained from TS3-Re-endo. ${ }^{\mathrm{c}}$ (3R,5S) obtained from TS3-Re-exo. ${ }^{\mathrm{d}}$ (3S,5S) obtained from TS3-Si-endo. e (3S,5R) obtained from TS3-Si-exo.

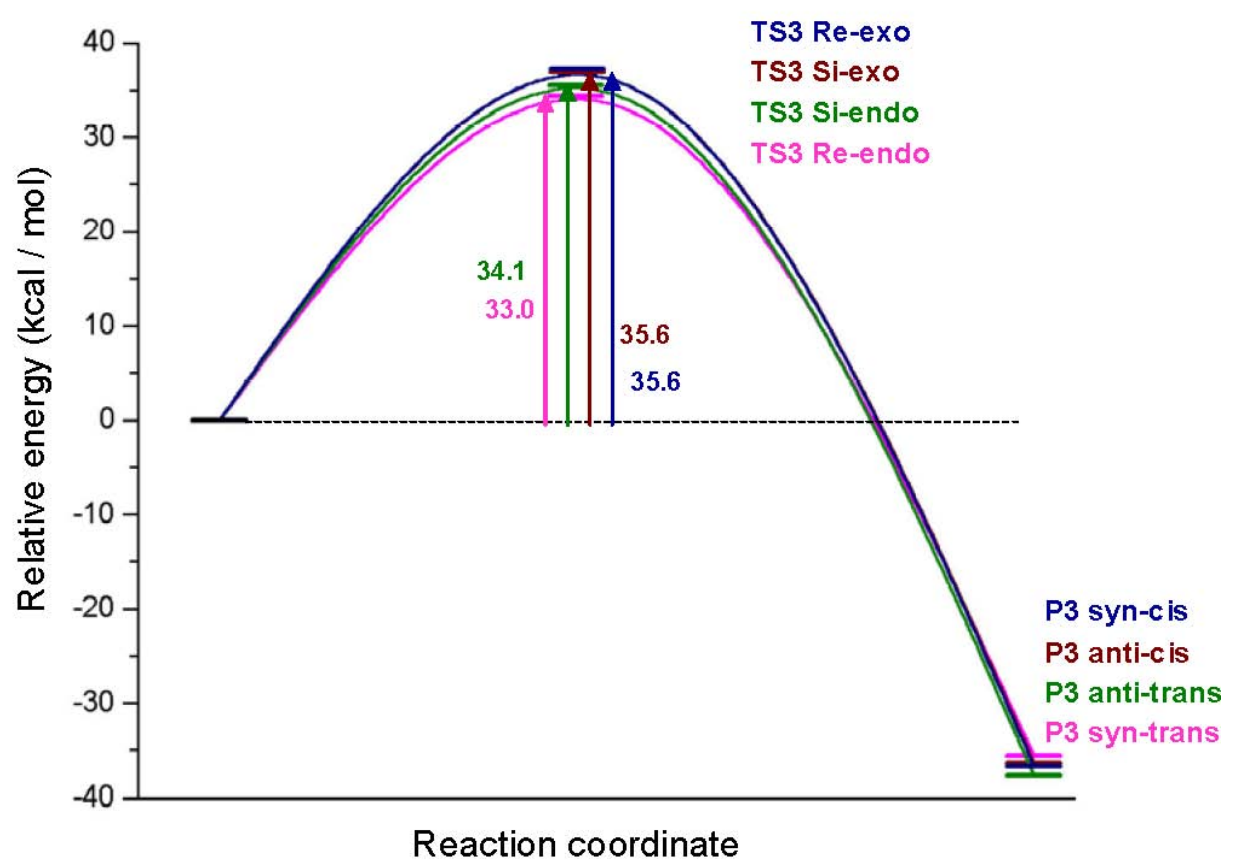

Figure 5. Energy profiles for the concerted addition of silyl enolates to nitrones in the absence of Lewis acids. Energy data correspond to those given in Table 2. 
This concerted mechanism may be considered as a plausible approach since it is possible to explain the formation of all the obtained products from the orthoester $\mathbf{5}$ by in situ desilylation induced by the Lewis acid that is present in the reaction medium (Scheme 6).
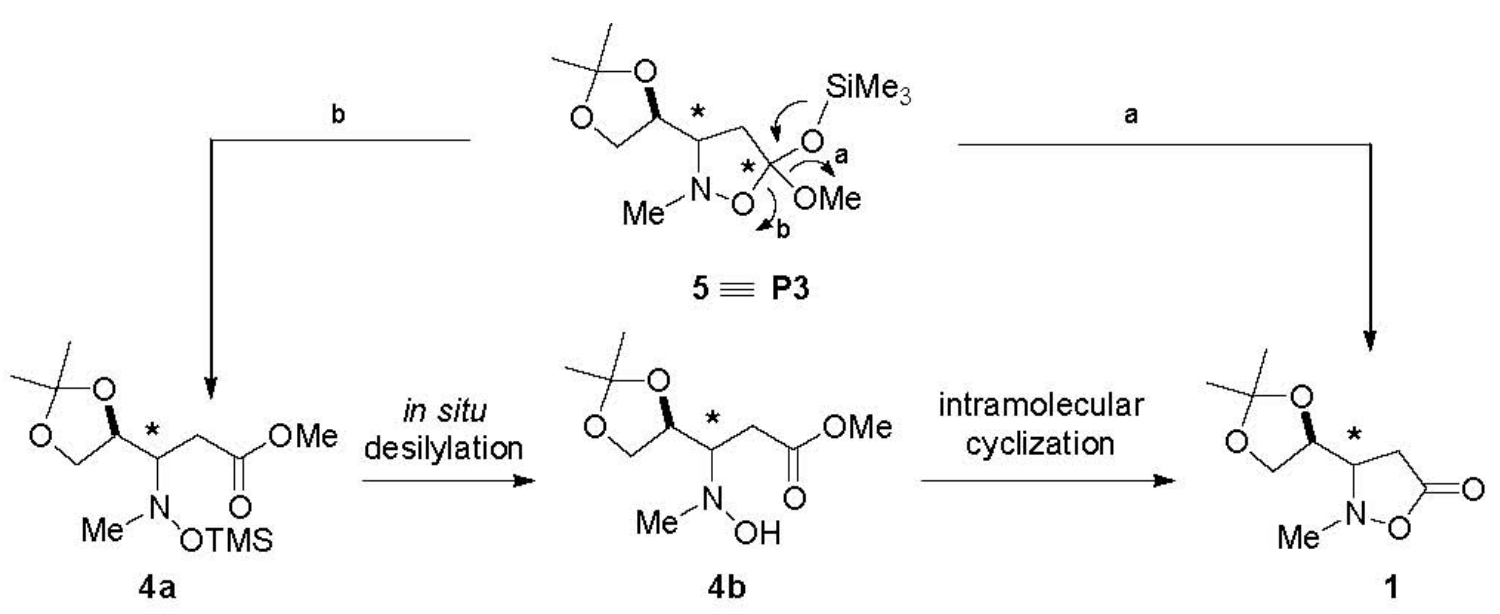

\section{Scheme 6}

Again, in this case the presence of an activating agent has not considered. Since it is proved experimentally that no reaction occur between $\mathbf{2}$ and $\mathbf{3}$ in the absence of Lewis acids, as it can be expected from the high energy barriers (more than $32 \mathrm{Kcal} / \mathrm{mol}$ ) this mechanism cannot be invoked for the studied reaction, yet.

We had reported that reactions with nitrone 2 in the absence of Lewis acids led to syn adducts in the case of nucleophilic addition processes, ${ }^{13}$ and, on the other hand, anti compounds were obtained in 1,3-dipolar cycloadditions. ${ }^{2 \mathrm{e}}$ At this point we think that this tendency could be applicable to the stereocontrolled addition of silyl ketene acetals to $\mathbf{2}$ and that two mechanisms may operate depending on the Lewis acid used as activating agent.

\section{With Lewis acids}

To rationalize the origin of the diastereoface discrimination introduced by the activating agent we decided to study the reaction of both TMSOTf and DEAC activated nitrones with silyl ketene acetal 3a. ${ }^{14}$ In principle, both stepwise and concerted mechanisms can be hypothesized. 


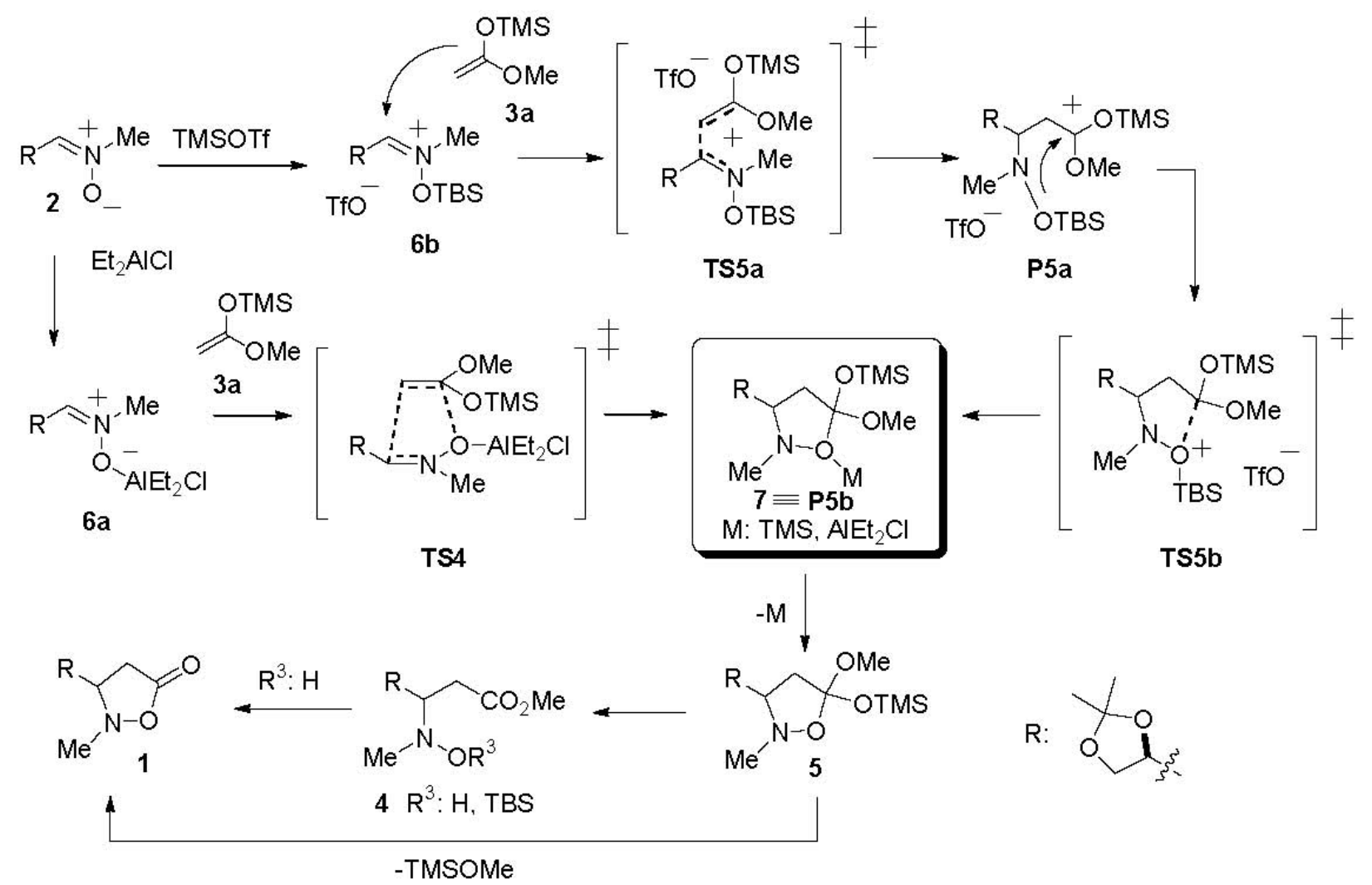

\section{Scheme 7}

The concerted one corresponds to a classical 1,3-dipolar cycloaddition. The stepwise mechanism has been previously proposed by Trombini et al. ${ }^{15}$ Scheme 7 highlights the sequence of steps that have been considered for each approach.

The exploration of the PESs for the reaction between silyl ketene acetal 3a and activated nitrones $\mathbf{6 a}$ and $\mathbf{6 b}$ rendered a concerted pathway for $\mathbf{6 a}$ and a stepwise pathway for $\mathbf{6 b}$. No transition states could be located neither for the concerted reaction of TMS-activated nitrone $\mathbf{6 b}$ nor for the stepwise reaction of DEAC-activated nitrone 6a. Moreover, starting from the located transition structures obtained for each reaction path (TS4 for the concerted one and TS5a and TS5b for the stepwise one), the alternative transition states were located when TMS and DEAC were exchanged. In other words, optimization of TS4 with the TS keyword after replacing DEAC by TMS led to TS5a, and vice versa.

\section{Concerted mechanism}

In a similar way to the concerted reaction studied above without Lewis acids, four transition states have been considered (Figure 6). The energy values for the stationary points and the 
energy profile for that concerted mechanism are given in Table 3 and Figure 7, respectively.

Table 3. Heats of formation (PM3) of the corresponding reagents, transition structures and products for the 1,3-dipolar cycloaddition of silyl ketene acetal 3a to $\mathbf{2}$ in the presence of DEAC

\begin{tabular}{ccc}
\hline & Energy (Kcal/mol) & Relative energy to reactants $^{\mathrm{a}}$ \\
\hline TS4-Re-endo & -245.5 & 14.6 \\
TS4-Re-exo & -247.1 & 13.0 \\
TS4-Si-endo & -248.4 & 11.7 \\
TS4-Si-exo $^{\mathrm{a}}$ & -249.7 & 10.4 \\
P4-syn-trans $^{\mathrm{b}}$ & -317.4 & -57.3 \\
P4-syn-cis $^{\mathrm{c}}$ & -319.7 & -59.6 \\
P4-anti-trans $^{\mathrm{d}}$ & -315.4 & -55.3 \\
P4-anti-cis $^{\mathrm{e}}$ & -317.0 & -56.9 \\
\hline
\end{tabular}

a The energy of the reactants has been calculated as the sum of that of the nitrone, dimetylaluminium chloride and the silyl ketene acetal, i.e.: $-260.1 \mathrm{Kcal} / \mathrm{mol} .{ }^{\mathrm{b}}$ (3R,5R) obtained from TS4-Re-endo. ${ }^{\mathrm{C}}$ (3R,5S) obtained from TS4-Re-exo. ${ }^{\mathrm{d}}$ (3S,5S) obtained from TS4-Si-endo. e (3S,5R) obtained from TS4-Si-exo. Compounds $\mathbf{P 4}$ correspond to 7 ( $\left.\mathrm{M=}=\mathrm{Et}_{2} \mathrm{AlCl}\right)$ in Scheme 6.

The barrier heights for all TS's are lower (10.4-14.6 Kcal/mol) that those corresponding to the reaction in the absence of Lewis acids (33.0-35.6, see Table 2), thus confirming the activation of the nitrone with aluminium. 

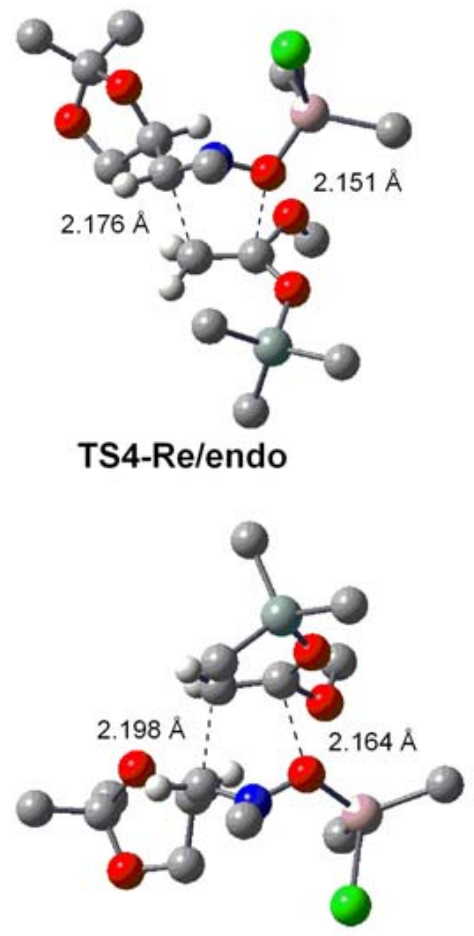

TS4-Si/endo

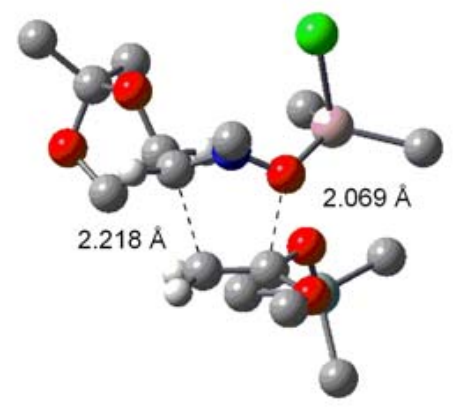

TS4-Re/exo

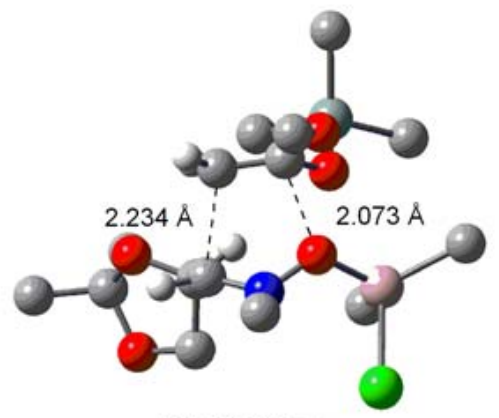

TS4-Si/exo

Figure 6. Transition states for the concerted reaction between 2 and $\mathbf{3 a}$ in the presence of DEAC.

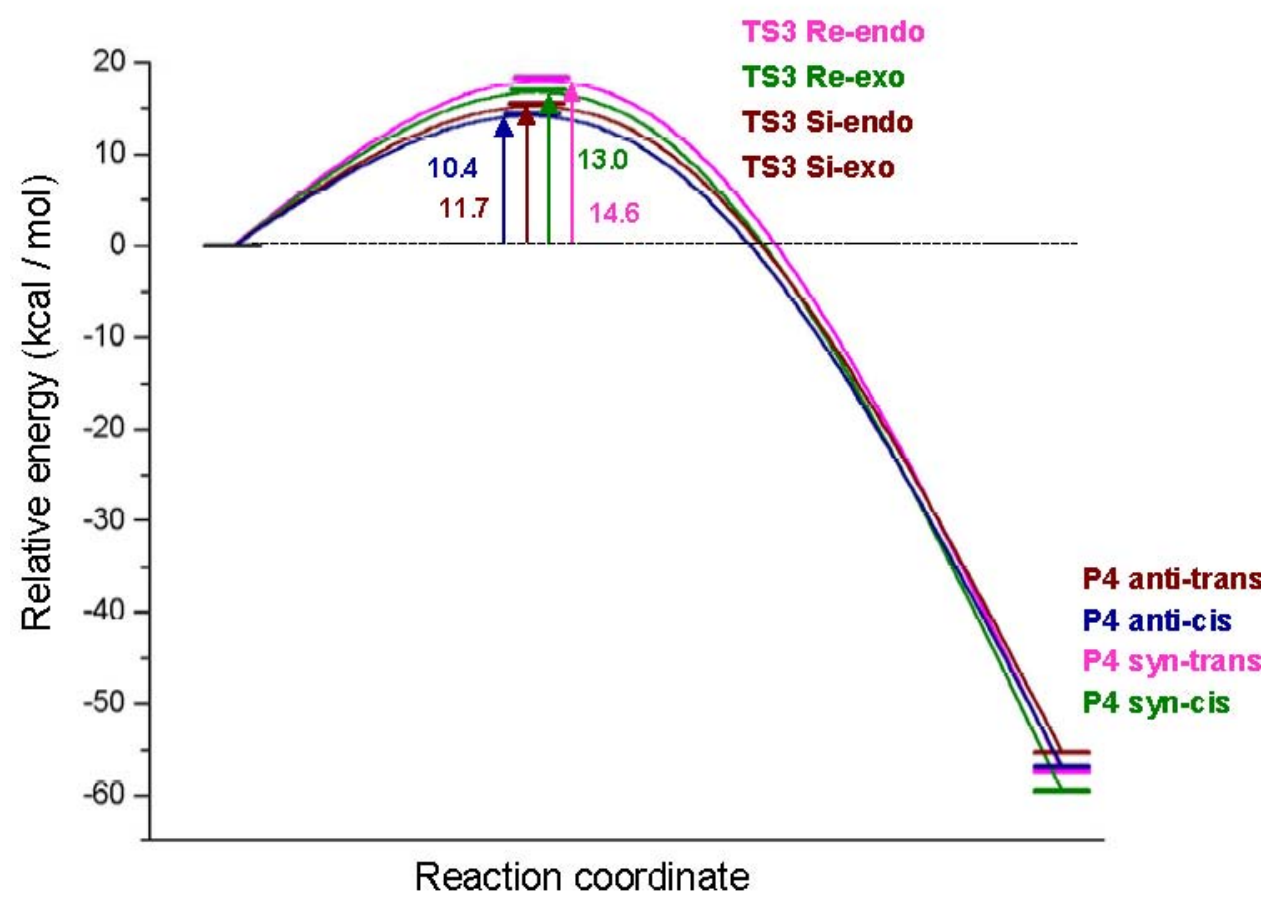

Figure 7. Energy profiles for the concerted addition of silyl enolates to aluminium-activated nitrones. Energy data correspond to those given in Table 3. 
From the stereochemical point of view, the attack by the Si face (leading to the anti adducts) is more favored than that by the Re face, thus predicting the preferential formation of anti adducts. This result, which is in agreement with the experimental data, is in a marked contrast with that obtained in the absence of Lewis acids, which predicted the syn adduct (Re attack) to be predominant.

\section{Stepwise mechanism}

The stepwise mechanism starts with the nucleophilic addition of enolate $3 \mathbf{a}$ to activated nitrone 6b. After exploration of the PESs for Re and Si attacks, leading to syn and anti adducts respectively, two transitions states were located (Figure 8).

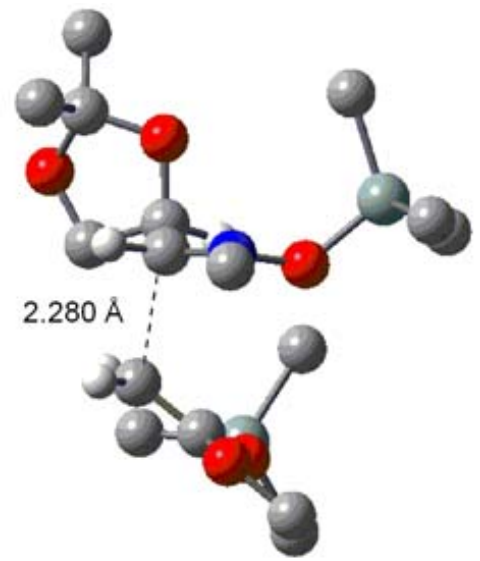

TS5a-Re

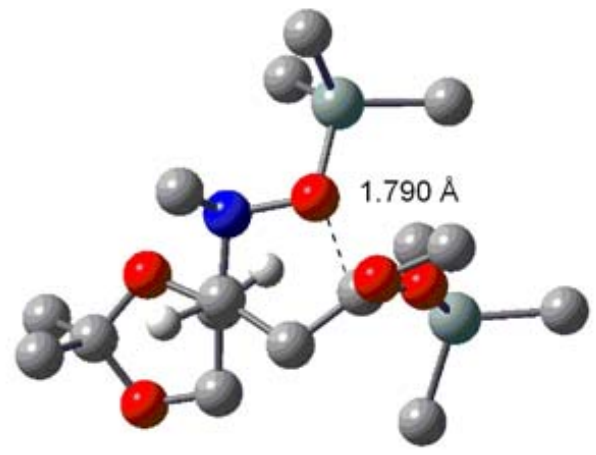

TS5b-Re

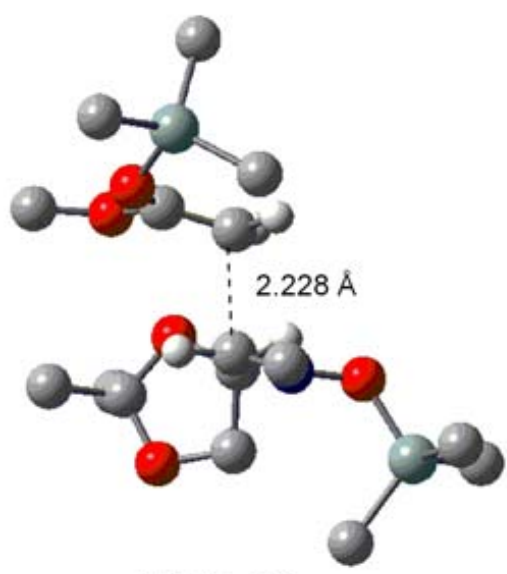

TS5a-Si

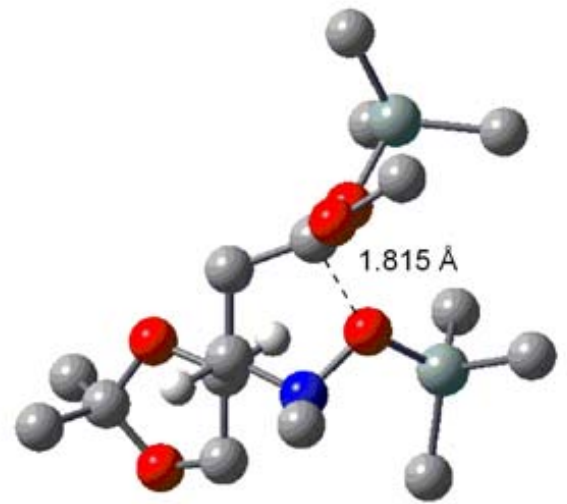

TS5b-Si

Figure 8. Transition states for the stepwise reaction between $\mathbf{2}$ and $\mathbf{3 a}$ in the presence of TMSOTf.

The IRC analysis of these transition structures showed the formation of two carbocationic 
intermediates P5a-syn and P5a-anti. A close inspection of the structures of these intermediates shows that they can be considered as ionic pairs. In fact, these intermediates lead to the final adducts through the corresponding transition states TS5b, and two different attacks, corresponding to the diastereotopic faces of the carbocation, can be considered for each intermediate. However, only TS5b-Re and TS5b-Si could be located (Figure 8); this observation can be attributed to the fact that, due to the proximity of the incoming oxygen atom (formation of a ionic pair), no bond rotation in the carbocation is allowed. This hypothesis is supported by the distances observed between the oxygen atom and the carbocation in the intermediates P5a (not shown in Figure 8) (2.543 $\AA$ for P5a-syn and $3.008 \AA$ for P5a-anti), and those calculated in the transition structures TS5b (1.790 $\AA$ for TS5b-Re and $1.815 \AA$ for TS5b-Si). The energies of all stationary points that connect them are given in Table 4. The energy profile starting from the silicon-activated nitrone is given in Figure 9.

Table 4. Heats of formation (PM3) of the corresponding reagents, transition structures and products for the stepwise nucleophilic addition of silyl ketene acetal 3a to $\mathbf{2}$ in the presence of TMSOTf

\begin{tabular}{ccc}
\hline & Energy (Kcal/mol) & Relative energy to reactants $^{\mathrm{a}}$ \\
\hline TS5a-Re & -134.7 & 5.9 \\
TS5a-Si & -132.6 & 7.9 \\
P5a-syn & -163.6 & -23.0 \\
P5a-anti & -161.5 & -20.9 \\
TS5b-Re-endo & -159.4 & $-18.8(4.2)^{\mathrm{g}}$ \\
TS5b-Re-exo & $------{ }^{\mathrm{f}}$ & \\
TS5b-Si-endo & -152.5 & $-11.9(9.0)^{\mathrm{h}}$ \\
TS5b-Si-exo & $------{ }^{\mathrm{f}}$ & \\
P5b-syn-trans $^{\mathrm{a}}$ & -164.1 & $-23.5(0.5)^{\mathrm{g}}$ \\
P5b-syn-cis $^{\mathrm{c}}$ & -164.9 & $-24.3(1.4)^{\mathrm{g}}$ \\
P5b-anti-trans $^{\mathrm{d}}$ & -164.0 & $-23.4(2.5)^{\mathrm{h}}$ \\
P5b-anti-cis $^{\mathrm{e}}$ & -161.6 & $-21.0(0.1)^{\mathrm{h}}$ \\
\hline
\end{tabular}

a Since the triflate counteranion has not been considered in the calculations, the energy of the reactants has been taken from the last point of the corresponding IRC calculation, after optimization without any restriction i.e.: $-140.6 \mathrm{Kcal} / \mathrm{mol} .{ }^{\mathrm{b}}$ (3R,5R) obtained from TS5b-Reendo. ${ }^{c}$ (3R,5S) obtained from TS5b-Re-exo. ${ }^{\mathrm{d}}$ (3S,5S) obtained from TS5b-Si-endo. e (3S,5R) obtained from TS5b-Si-exo. ${ }^{\mathrm{f}}$ The corresponding transition structure was not found. ${ }^{\mathrm{g}}$ relative to 
P5a-syn. helative to $\mathbf{P 5 a - a n t i . ~ C o m p o u n d s ~} \mathbf{P 5 b}$ correspond to $7\left(\mathbf{M}=\mathrm{SiMe}_{3}\right)$ in Scheme 6.

The first step of the reaction, corresponding to the formation of intermediates P5a is the limiting step. The second one can also be seen as a valence tautomerism between P5a and 7 $\left(\mathrm{M}=\mathrm{SiMe}_{3}\right)$. In fact, the potential surface connecting these two points is rather flat as can be concluded from the calculations (see relative energies in Table 4). ${ }^{16}$ The lower barrier heights (5.9-7.9 Kcal/mol) found for the limiting first step explain the experimentally observed increased reactivoity for nitrone activated with silyl triflate. Again, these lower values are in contrast with those found for the reaction in the absence of any additive (see Table 2).

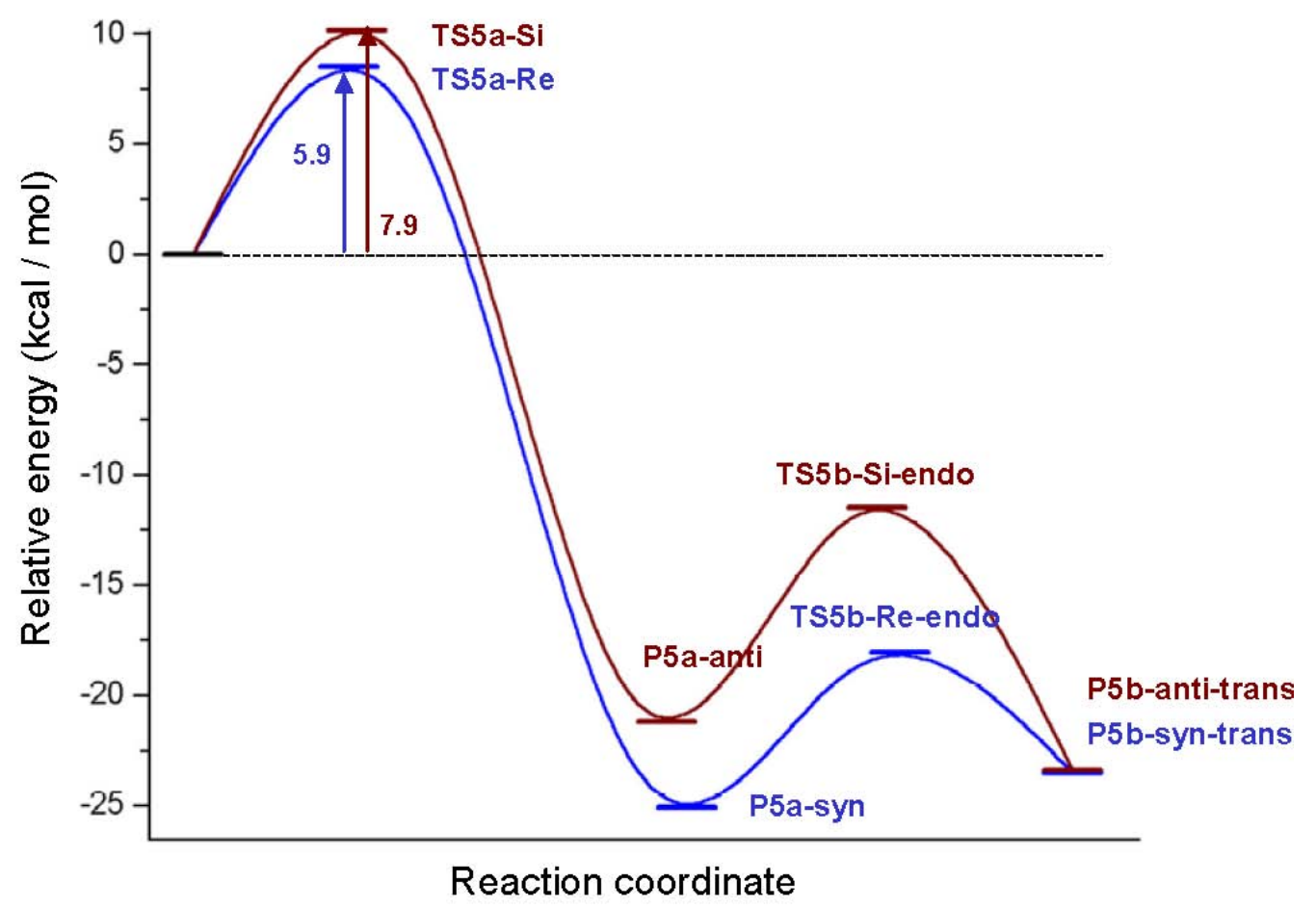

Figure 9. Energy profiles for the stepwise addition of silyl enolates to silicon-activated nitrones. Energy data correspond to those given in Table 4.

\section{Conclusions}

Several mechanisms for the addition of silyl ketene acetals $\mathbf{3}$ to chiral nitrones $\mathbf{2}$ have been studied at a semiempirical level (PM3) due to both the size of the molecules and the magnitude of the study. Both concerted and stepwise processes can be invoked, but only when Lewis acids are considered the theoretical calculations are concordant with experimental results. In such 
cases, the predicted stereochemistry is on good agreement, from a qualitative point of view, with the experimental results. The addition of silyl ketene acetals to chiral nitrones derived from Dglyceraldehyde in the presence of trimethylsilyltrifluoromethane sulfonate is best described as a nucleophilic addition reaction, while the corresponding addition in the presence of diethylaluminium chloride is assigned to be a concerted reaction.

\section{Acknowledgements}

Prof. L.R. Domingo (University of Valencia, Spain) is gratefully acknowledged for helpful discussions. This research is supported by grants from the Diputacion General de Aragon, Spain (Project P079/99-C) and the Ministerio of Educacion y Cultura (Project PB97-1014). One of us (J.A.M.) thanks the Diputacion General de Aragon for a fellowship.

\section{References and Notes}

1. Frederickson, M. Tetrahedron 1997, 53, 403-425.

2. (a) Chiachio, U.; Rescifina, A.; Corsaro, A.; Pistara, V.; Romeo, G.; Romeo, R. Tetrahedron: Asymmetry 2000, 11, 2045. (b) Chiacchio, U.; Corsaro, A.; Gumina, G.; Rescifina, A.; Iannazzo; D.; Piperno, A.; Romeo, G.; Romeo, R. J. Org. Chem. 1999, 64, 9321. (c) Xiang, Y.; Gong, Y.; Zhao, K. Tetrahedron Lett. 1996, 37, 4877. (d) Xiang, Y.; Gi, H.-J.; Niu, D.; Schinazi, R.F.; Zhao, K. J. Org. Chem. 1997, 62, 7430. (e) Merino, P.; Alamo, E.M.; Franco, S.; Merchan, F.L.; Simon, A.; Tejero, T. Tetrahedron: Asymmetry 2000, 11, 1543. (f) Merino, P.; Franco, S.; Garces, N.; Merchan, F.L.; Tejero, T. Chem. Commun. 1998, 493.

3. For a review see: Pan, S.; Amankulor, N.M.; Zhao, K. Tetrahedron 1998, 54, 6587.

4. (a) Merino, P.; Franco, S., Merchan, F.L.; Tejero, T. Tetrahedron: Asymmetry 1998, 9, 3945. (b) Merino, P.; Franco, S., Merchan, F.L.; Tejero, T. Tetrahedron Lett. 1998, 39, 6411. (c) Merino, P.; Franco, S., Merchan, F.L.; Tejero, T. J. Org. Chem. 2000, 64, 5575.

5. (a) Saha, N.N.; Desai, V.N.; Dhavale, D.D. Tetrahedron 2001, 57, 39. (b) Jost, S.; Gimbert, Y.; Greene, A.E.; Fotiadu, F. J. Org. Chem. 1997, 62, 6672. (c) Kita, Y.; Itoh, F.; Tamura, O.; Ke, Y.Y.; tamura, Y. Tetrahedron Lett. 1987, 28, 1431. (d) Kita, Y.; Tamura, O.; Itoh, F.; Kishino, H.; Miki, T.; Kohno, M.; Tamura, Y. Chem. Commun. 1988, 761.

6. Qian, C.; Wang, L. Tetrahedron 2000, 56, 7193. 
7. (a) Merino, P.; Alamo, E.M.; Bona, M.; Franco, S.; Merchan, F.L.; Tejero, T.; Vieceli, O. Tetrahedron Lett. 2000, 41, 9239. (b) Merino, P.; Franco, S. unpublished results.

8. Stewart, J.J.P. In Reviews in computational chemistry; Lipkowitz, K.B.; Boyd, D.B., Eds; VCH: New York, 1990; pp. 45-81.

9. MOPAC package was used as implemented in WINMOPAC 2.0. Fujitsu Ltd.

10. Stewart, J.J.P. J. Comput. Chem. 1989, 10, 20.

11. Fukui, K. J. Phys. Chem. 1970, 74, 4161.

12. Two molecules of water were included in the calculation in order to complete the coordination sphere of the lithium atom.

13. For an account see: Merino, P.; Franco, S.; Merchan, F.L.; Tejero, T. Synlett 2000, 424.

14. For recent theoretical studies on nitrones complexes with Lewis acids see: (a) Tanaka, J.; Kanemasa, S. Tetrahedron 2001, 57, 899. (b) Domingo, L.R. Eur. J. Org. Chem. 2000, 2265.

15. (a) Camiletti, C.; Dhavale, D.D.; Gentilucci, L.; Trombini, C. J. Chem. Soc. Perkin Trans 1. 1993, 3157. (b) Camiletti, C.; Dhavale, D.D.; Donati, F.; Trombini, C. Tetrahedron Lett. 1995, 36, 7293. (c) Dhavale, D.D.; Trombini, C. Chem. Commun. 1992, 1269.

16. The term valence tautomerism consists of an equilibrium between two different structures in which only electrons shift, the position of the atoms not being the same (so, it is rather different of resonance). For illustrative examples of valence tautomers and an explanation of the concept see: March, J. Advanced Organic Chemistry, $4^{\text {th }}$ Edn; John Wiley: New York. 1992. pp 1134-1136. 